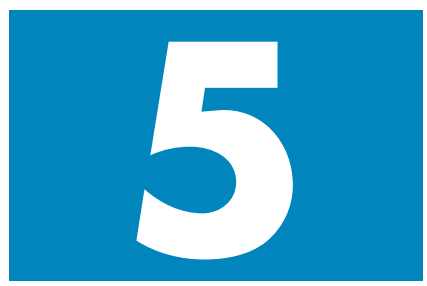

\title{
Good occlusal practice in removable prosthodontics
}

\author{
S. J. Davies, ' R. M. J. Gray, ${ }^{2}$ and J. F. McCord, ${ }^{3}$
}

\begin{abstract}
The loss of teeth may result in patients experiencing problems of a functional, aesthetic and psychological nature.

This section addresses the very important subject of occlusal considerations for partial and complete dentures. The occlusion is particularly important given the bearing that occlusal factors have, especially on edentulous patients.
\end{abstract}

In this part, we will discuss:

- The features of an ideal occlusion in removable prosthodontics

- Why these features make it 'ideal' for denture stability

- Introduce some techniques for achieving these aims
$1^{*}$ GDP, 73 Buxton Rd, High Lane, Stockport SK6 8DR; P/T Lecturer in Dental Practice, University Dental Hospital of Manchester, Higher Cambridge St., Manchester M15 6FH; ${ }^{2}$ Honorary Fellow, University Dental Hospital of Manchester, Higher Cambridge St., Manchester M15 6FH ${ }^{3}$ Professor of Restorative Dentistry, Unit of Prosthodontics, University Dental Hospital of Manchester, Higher Cambridge St, Manchester M15 6FH ${ }^{\star}$ Correspondence to: Stephen Davies email:stephen.j.davies@man.ac.uk REFEREED PAPER

(C) British Dental Journal 2001; 191: 491-502
$\mathrm{H}$ istorically complete denture prosthodontics has been at the forefront of the study of occlusion and many of the terms used in occlusion have their origin in this subject. The reason that occlusion has always been a consideration in the provision of removable complete prosthetics is because the adoption of good occlusal practice has a significant and immediate impact on the overall success of the treatment, as it affects denture stability. If an inappropriate occlusion is built into a denture then the patient will be unlikely to be able to accommodate to that denture and the dentist will be immediately aware that the treatment has been unsuccessful. The reason why the correct distribution of occlusal forces is so important in the design of removable prosthetics is because the prosthetic teeth that provide the occlusion are not directly attached to the patient.

Students of occlusion have good reason to be grateful to the science of prosthodontics and it remains a part of the undergraduate course where clear guidance on 'good occlusal practice' will be available.

Terminology (see box below)

Terms like 'non-working side' or the more accurate term in a prosthetic sense 'balancing side' are based in a study of occlusion from the perspective of complete dentures and refer to the side of the dentures which are not being used for chewing during a lateral excursion. This can lead to confusion when considering the temporomandibular joints during that same lateral excursion because the 'non-working side' joint is moving much more than the one on the 'working side'. The terms 'Centric Relation' and 'Centric Occlusion' will be used instead of their synonyms of 'Retruded Contact Position and Intercuspation Position'. Additionally the term 'Static Occlusion' will be used to describe occlusal contacts when the mandible is closed and still, and the term 'Dynamic Occlusion' will be used to describe occlusal contacts when the mandible is moving. These terms will be used in preference to the 'prosthetic' terms of 'occlusion' and 'articulation'.

\section{Classification}

Prostheses are often considered under the categories of partial or complete dentures, but partial dentures may be supported by teeth, mucosa or a combination of both and, given the fact that the nature of that support dictates the design of the ideal occlusal platform, partial dentures are divided into the following sections:

- Tooth-supported dentures

- Tooth and mucosa-supported dentures

- Mucosa-supported dentures.

\section{Tooth-supported dentures}

The concept of the occlusal prescription being 'conformative' or 're-organised' has been discussed previously. The former conforms to the constraints of the patient's present occlusal scheme while the latter alters or re-organises the current scheme to a more idealised occlu-

$$
\begin{array}{ll}
\text { Terminology } & =\text { Retruded Contact Position (RCP) } \\
\text { Centric Relation (CR) } & =\text { Intercuspation Position (ICP) } \\
\text { Centric Occlusion (CO) } & =\text { Balanced Occlusion } \\
\text { Balanced Static Occlusion } & =\text { Balanced Articulation }
\end{array}
$$




\section{In a denture \\ supported by \\ teeth: \\ - The occlusion is \\ sensed \\ - The prostheses is \\ less likely to cause instability, if poorly designed}

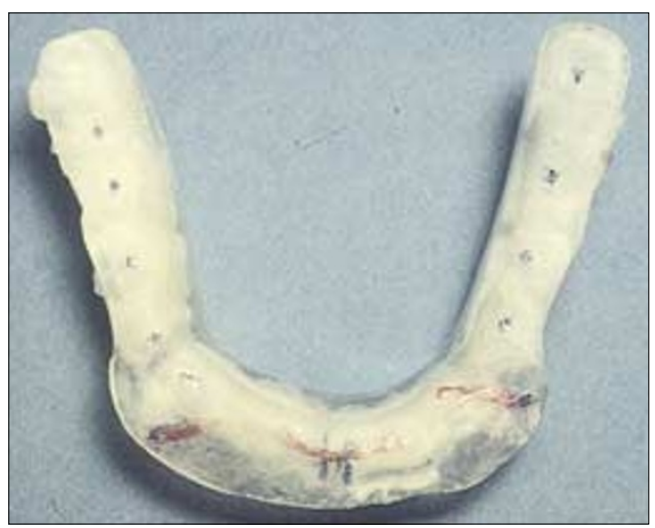

Fig I Occlusal diagnostic appliance (stabilisation splint)

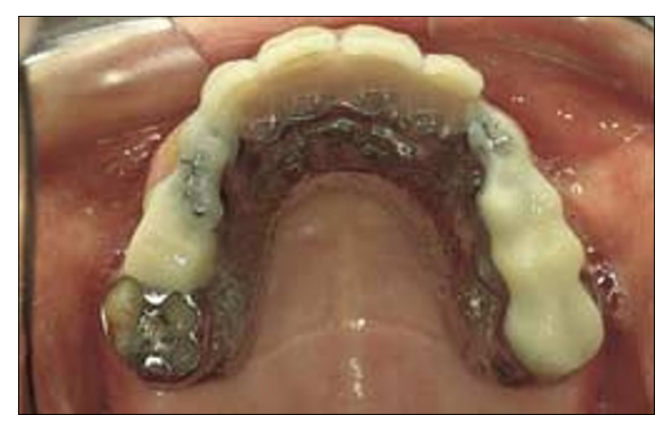

Fig 2 Hybrid prosthesis

sion, sometimes at a raised occlusal vertical dimension. If a partial denture is tooth supported then the design of the occlusion provided by that prosthesis should be to 'conform' with and be complementary to the existing occlusion. The only exceptions to this would be 'rehabilitative' prostheses, which are occlusal diagnostic splints (Fig. 1) and hybrid prostheses (essentially overdentures) (Fig. 2). The difference between the two is that the splint is not intended to be definitive whereas the hybrid prostheses is. Conventional wisdom indicates that the dentist must determine the patient's ability to withstand a raised occlusal vertical dimension with a temporary (diagnostic) prosthesis prior to prescribing the definitive denture/prosthesis.

If a partial denture is totally tooth-supported the patient's occlusion is entirely borne by teeth. This has two important occlusal consequences. Firstly it means that occlusal load will

\section{The EDEC principle:}

$$
\begin{aligned}
& \mathbf{E}=\text { Examine the pre-existing condition } \\
& \mathbf{D}=\text { Design the prosthesis } \\
& \mathbf{E}=\text { Execute the prosthesis } \\
& \mathbf{C}=\text { Check the occlusion at completion }
\end{aligned}
$$

be 'sensed' by the proprioceptors of the periodontal membranes. Secondly, it is less likely that a poorly designed occlusion will be immediately manifested by the denture being unstable than would be the case if the support was in part or totally mucosa supported.

\section{Examination}

The most important aspect of the examination of the patient, for whom a tooth-borne partial denture is to be made, is to confirm that it will indeed be solely supported by the existing teeth, and not in part by the soft tissues. It is advisable, as in all patients for whom a treatment is envisaged, to carry out a comprehensive examination not only of the dental and periodontal tissues, but also of the articulatory system.

\section{The EDEC principle}

\section{$E=$ Examination}

The examination of the patient's pre-treatment occlusion is the first stage.

Treatment

\section{$D=$ Design}

The support for the partial denture must be provided by the abutment teeth in such a way as to avoid a change in the occlusal contact of the other teeth, otherwise the treatment would not be within the conformative approach. Ideally the rests should also be designed to transfer the occlusal load down the abutment teeth along their long axes.

\section{$E=$ Execute}

The design criteria as expressed above may require modification of the abutment teeth and/or the opposing teeth. If this is the case, a clear rationale for the changes can be presented to the patient, and is likely to be much better received than alteration to the dentition after or at the fit of the prosthesis.

\section{$C=$ Check}

At the delivery stage, a check is made that the prosthesis has added to the patient's occlusal platform rather than altered its position or dynamic occlusal characteristics. This is easily achieved providing a record of the patient's pre-treatment static and dynamic occlusal contacts has been recorded. It does not matter whether this is a three-dimensional record such as mounted study models on a semiadjustable articulator, or a two-dimensional record such as a written record or 'occlusal sketch' of articulating paper marks. It is obviously impossible to confirm that the treatment has been provided within the conformative approach unless this pre-treatment record exists. 


\section{Tooth and mucosa-supported dentures/ prostheses}

The mucosa under a denture is capable of displacement to a degree that is 20 times greater than that of teeth via the periodontal membrane. ${ }^{1}$ Furthermore there is a difference not only in the amount of displacement under load but also in the type of deformation. The periodontal membrane of teeth, under occlusal force, undergoes a simple elastic deformation whereas mucosa undergoes visco-elastic deformation. This means that the recovery from mucosal deformation is more prolonged than that of a tooth in its socket. These quantitative and qualitative differences between tooth support and mucosa support have an important clinical significance; and obviously could cause problems in the construction of a prosthesis in which the occlusal load is going to be shared between what are two very different tissues.

\section{Examination}

The examination of a patient's mouth before the provision of a tooth/mucosa supported removable prosthesis is designed to assess the support, retention and stability provided by the teeth, the ridges and the mucosa. A simple chart is suggested as an aid to this objective (Chart 1).

For a patient with favourable prospects for support, retention and stability of their tooth/ ridge/mucosal tissues the chart may appear as in Chart 2. Patient expectations can be better managed if a chart similar to this is used as part of the examination phase

\section{Treatment}

The principal consequence of occlusal loading onto the more deformable mucosa will be the loss of occlusal contact. This is a particular problem in patients with free-end saddles. It was for this reason that Applegate described a technique of denture construction, ${ }^{2}$ universally known as the 'altered cast technique', which consists of the following stages:

1. Following the recording of the definitive impression, the metal framework is cast and tried-in. If satisfactory, the saddle area(s) is(are) covered with light-cured denture base material.

2. The base of the saddle areas is on-laid with light-bodied impression material and an impression recorded of the saddle area with the dentist pressing on the tooth-borne elements of the framework.

3. The original master cast is sectioned at the distal abutments and the saddles areas discarded. The new saddle area(s) are prepared by pouring from this new impression.

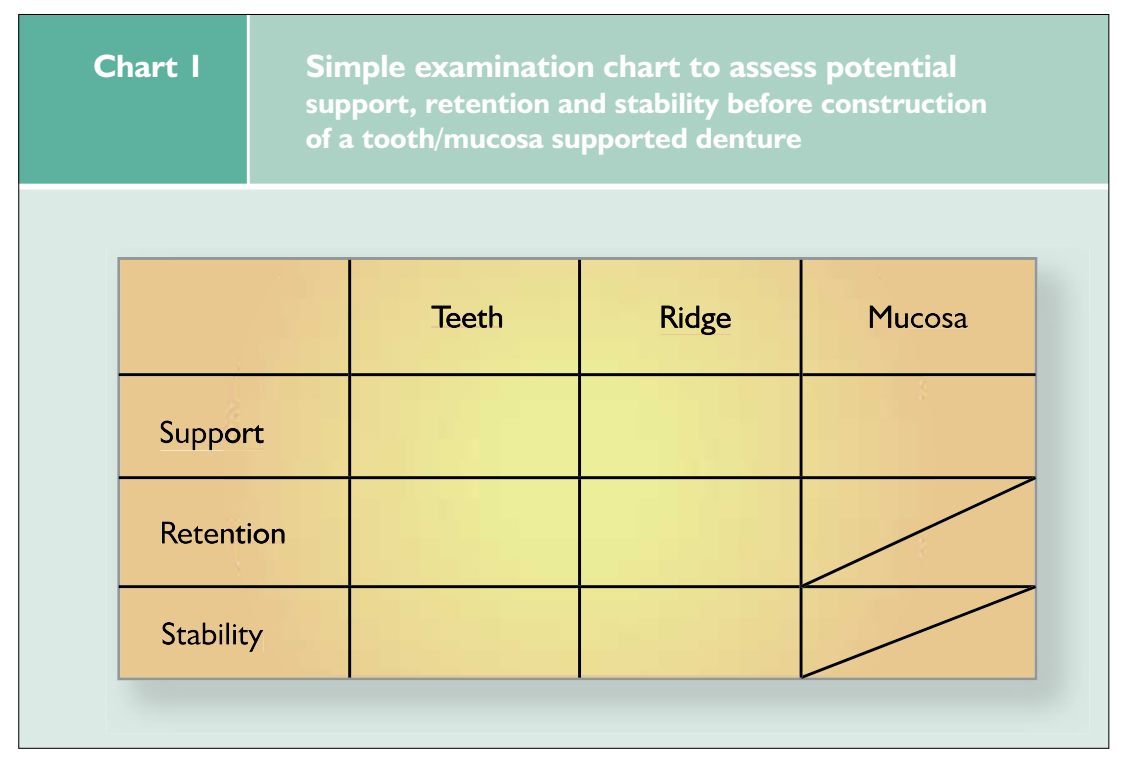

\begin{tabular}{|l|c|c|c|}
\hline & Teeth & Ridge & Mucosa \\
\hline Support & good perio. & square & firm \\
\hline Retention & good undercuts & favourable & \\
\hline Stability & good & favourable & \\
\hline
\end{tabular}

The intention of this technique is to ensure that the occlusal pressure will still be resisted by the ridges after the natural teeth have been minimally displaced into their sockets.

An identical clinical procedure may be undertaken for a reline and this maybe sufficient to restore the occlusion in saddle areas.

\section{Mucosa-supported dentures}

\section{Partial dentures}

Dentists should be under no illusion that mucosally-supported partial dentures will, within a relatively short time, lose occlusal contact with the opposing arch as the underlying bone is resorbed; this type of denture cannot be relied upon to provide a lasting occlusion. In addition, the problem implied in the term 'gum strippers' is well known; a typical example is shown in Figure 3 illustrating the iatrogenic effects of selecting a mucosally-supported design. Simple relines are only likely to exacerbate the resorption.

However, not all such designs are necessarily examples of poor dentistry; for instance, train- 


\section{Fig 3 Gum stripper}

Fig 4 Training denture

For many patients, a simple occlusal prescription is all that will be required, ie the patient has 'evolved' to using essentially vertical mandibular movements with little or no lateral and protrusive mandibular movements.

In this case, no elaborate occlusal scheme is indicated, nor is a semi-adjustable articulator, because the dynamic occlusion can be ignored. act with dentures as with natural teeth. ${ }^{3}$ It is unlikely that this can be achieved, but it remains the goal.

The design of an occlusion in complete dentures is different from that of the dentate patient. While both are concerned with the final act of intermaxillary closure, the absence of direct attachment between the dentures and the patient's musculo-skeletal system requires a different set of guidelines of good occlusal practice.

For all these reasons, it is important to consider the role of occlusion in complete denture philosophy. The fundamental philosophies governing the biomechanics of complete dentures state that there is a fine inter-relationship between support, retention and stability, and the success of the prosthesis will be dependent in a very large part on these features. Importantly occlusion is considered a major factor governing stability. ${ }^{4}$

The minimal level of occlusion that any practitioner should prescribe in complete dentures is balanced occlusion; this is described by the British Society for the Study of Prosthetic Dentistry ${ }^{5}$ as ' even, harmonious bilateral contact between teeth or tooth analogues in retruded contact position (RCP)'. In our terminology this means a 'balanced centric occlusion in centric relation' $(\mathrm{CO}=\mathrm{CR})$. This is a 'static occlusion' concept and this type of an occlusion would ensure that, as a patient elevated the mandible into $C R$, the dentition would be stable. There would be no tilting/displacing force on the dentures and so stability would not be compromised. Factors

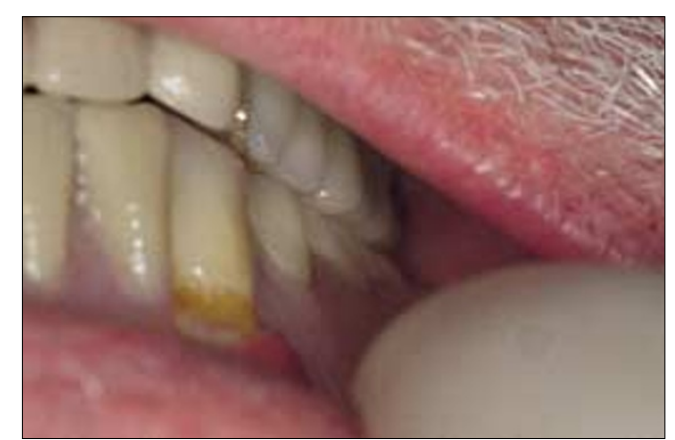

Fig 5 Occlusion at insertion

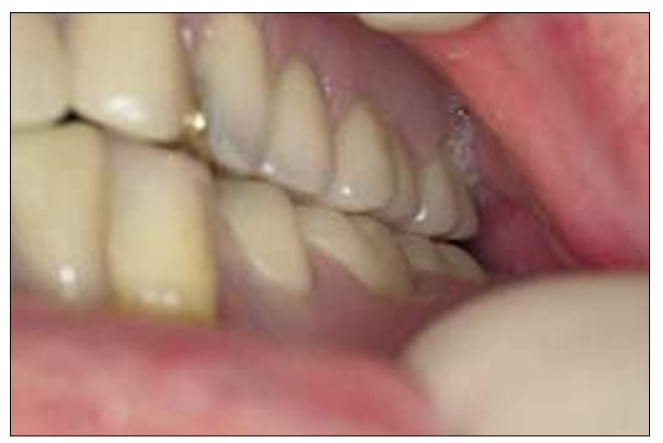

Fig 6 Lack of occlusion after 6 months 


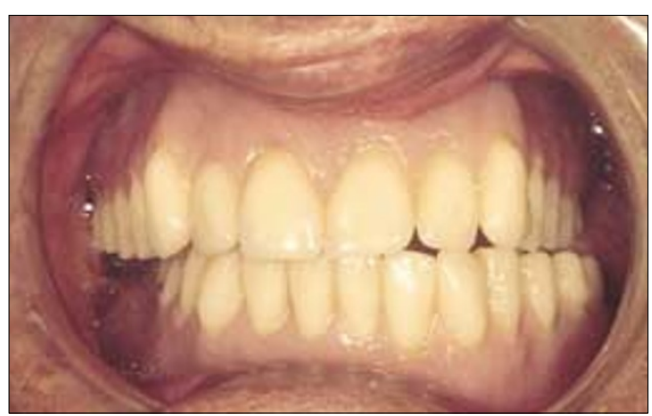

Fig 7a Unilateral prematurity

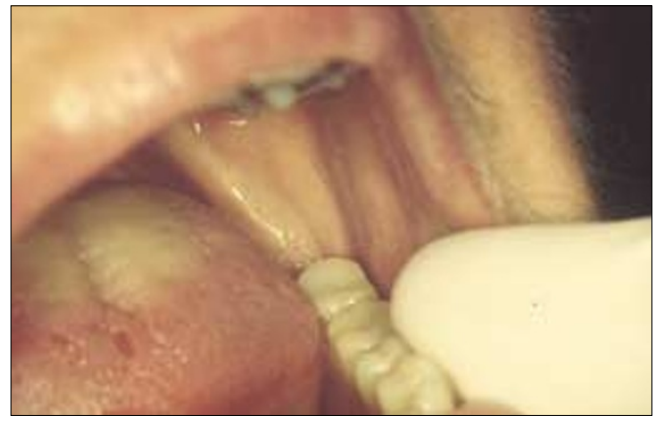

Fig 7c Injudicious placement of teeth

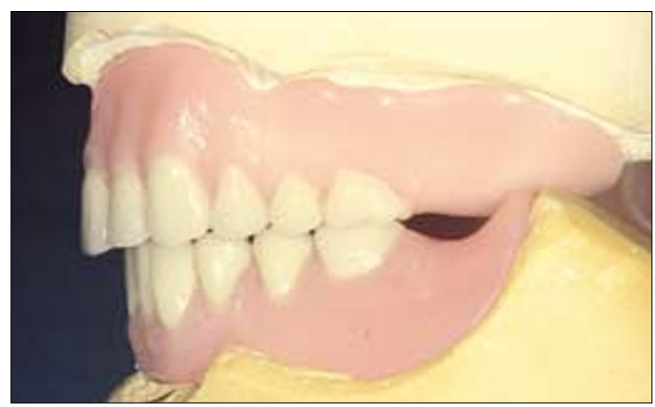

Fig 9a F/F with compensating curves

which might compromise this stability are illustrated in Figure 7. They are:

- Unilateral prematurities

- Occlusal tables which are too large

- Injudicious placement of teeth.

For other patients, however, lateral and protrusive movements are part of their normal 'ruminatory' mandibular pattern and for these patients, a balanced dynamic occlusion (balanced articulation) is required. In other words, consideration must be given not only to the static but also to the dynamic occlusal prescription.

In this situation, the teeth of the maxillary denture must maintain harmonious sliding contacts with the teeth of the mandibular denture in all excursive movements otherwise denture stability may be significantly compromised. For example, in a natural dentition, the act of protrusion usually results in a posterior open bite (the Christensen phenomenon Fig. 8). Such a situation would lead to instability in complete dentures, hence compensating

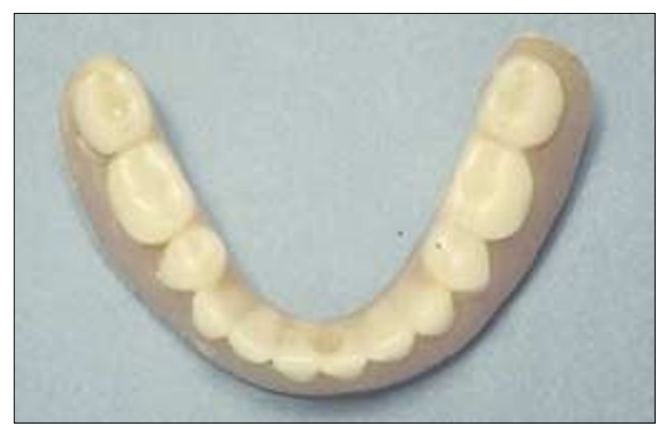

\section{Fig $7 b$ Too large an occlusal table}

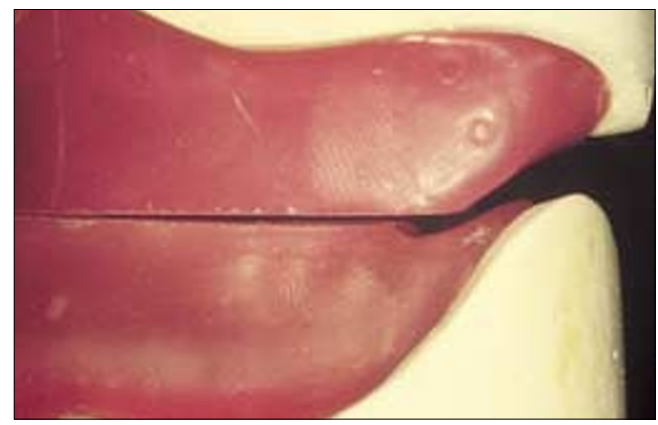

Fig 8 Christensen phenomenon

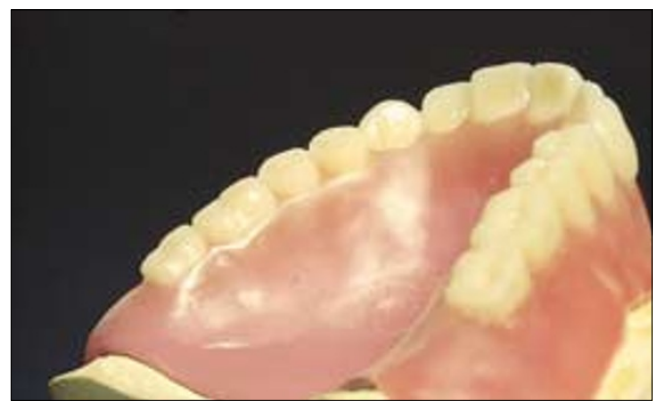

Fig 9b F/F with compensating curves

curves (Fig. 9a and b) are incorporated into the dentures. The same philosophy holds for lateral excursions.

This means that the 'ideal occlusion' for a patient with complete dentures differs from the 'ideal occlusion' for a dentate patient, for example it is 'ideal' for complete denture stability if there is no posterior disclusion during lateral excursions, whereas immediate and lasting posterior disclusion is usually considered to be ideal for the dentate patient.

It is because teeth on a denture are not attached to the patient's neuro-muscular skeletal system and there is no possibility of neural stimulation via periodontal proprioceptors that the criteria of what makes an 'ideal occlusion' have changed. Although there are mechanoreceptors in the denture bearing oral mucosa, they do not continue to send a stream of impulses to the sensory cortex.

It, therefore, beholds the dentist to determine the occlusal requirements of complete denture wearers prior to prescribing complete 
dentures. $^{6}$ If balanced articulation is required, there is no valid reason for not:

- Using a facebow

- Accurately determining condylar angles

- Harmonising the occlusion to match mandibular movements.

\section{Summary}

It is a prerequisite, for stability in all complete dentures that posterior occlusal contacts occur simultaneously and bilaterally; furthermore these contacts should occur in centric relation and at the appropriate occlusal vertical dimension. In some patients (those with a ruminatory chewing pattern) it will also be necessary to harmonise the dynamic occlusion in order to ensure denture stability.

Examination of the complete denture patient The purpose of the examination is to lead to the correct decisions being taken at each stage so that a successful treatment strategy can be made.

The examination of the denture bearing

surfaces

An assessment of the denture bearing surfaces will involve assessment of:

- Shape of the ridges: Moses suggested a classification of ridge shape and described the retentive and support characteristics of each (Plot 1). ${ }^{7}$ The ideal occlusion for the prosthesis will, therefore, relate to the ridge shape.

- Nature of denture bearing area: Firm or flabby; and sensitive or comfortable to finger pressure. A sensitive or mobile ridge will require an occlusal prescription that is designed to reduce the transmission of force.

- Space: If there is very little space between the ridges (usually at the posterior part of the mouth) then the distal extent of the occlusal platform will be necessarily reduced in length. It is better that the dentist discovers this at the examination stage rather than the technician when mounting the models.
The examination of the existing dentures Do the existing dentures exhibit?

- Inadequate freeway space

- Evidence of excessive wear: loss of vertical dimension, crossbite, and anterior posturing

If the answer to any of these questions is 'yes', then the new dentures are not going to be made to the 'conformative approach', but rather the occlusion will be changed ie the ' reorganised approach' will be adopted.

\section{The examination of how the patient masticates}

The answer to this essential question will in most cases determine whether the patient needs only a balanced static occlusion in centric relation, ('balanced occlusion'), or whether in addition they should have a balanced dynamic occlusion, ('balanced articulation').

In order to determine whether the patient has a chewing pattern that involves essentially only vertical mandibular movements, or also uses lateral and protrusive movements, they must be observed masticating.

This seems self evident, and yet very few patients are treated after determining, by observation, the type of chewing that is used. The appropriate occlusal prescription of the denture cannot be determined without this part of the examination which involves the use of the 'diagnostic biscuit' (Fig. 10).

\section{Treatment}

Treatment strategy

This will have evolved during the examination:

Should the conformative or re-organised approach be used?

In the vast majority of patients with a restorative/prosthetic need the conformative approach is the method of choice. It offers the dentist the relatively simple task of providing treatment which conforms to the occlusion to which the patient has been accustomed.

In the edentulous patient the re-organised approach may be indicated if the patient exhibits:

- A temporomandibular disorder

- A grossly overclosed vertical dimension

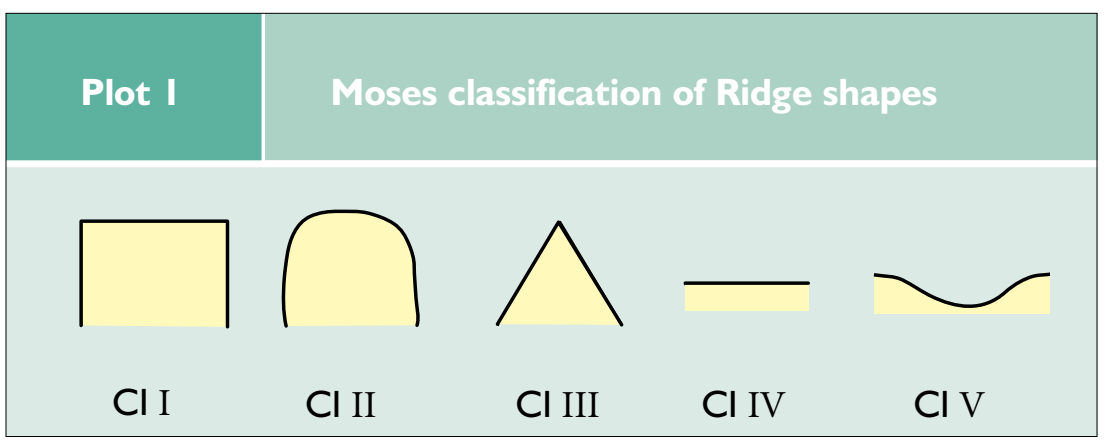


- A pre-existing inappropriate occlusal pattern that has led to denture instability.

If the re-organised approach is to be used; the next question is whether there is a need for a pre-treament phase of splint therapy. If so, copies of the patient's old dentures will be converted into splints that can be used to establish a new jaw relationship (vertical, horizontal or anterio-posteriorly).

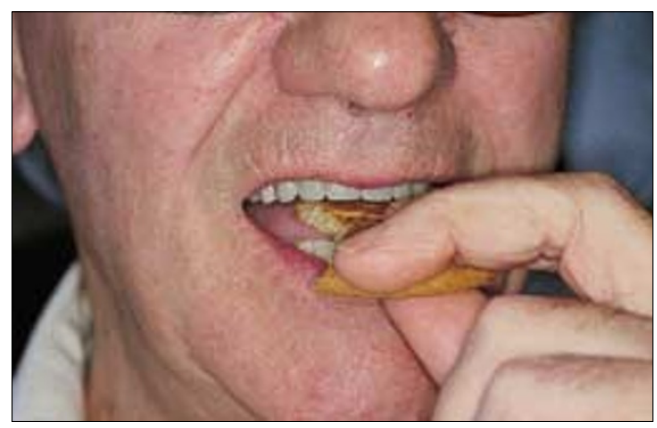

Fig 10 Diagnostic biscuit

Does the patient need a balanced dynamic occlusion (balanced articulation), or only a balanced static occlusion (balanced occlusion)?

This question can only be answered by determining, by observation, the type of mastication that the patient employs. This has major consequences for the treatment of the patient, and an assessment, therefore, of the patient chewing is an essential part of the examination of an edentulous patient.

\section{Stages of construction}

1. The design of the fitting surfaces 'impression taking'

It is the dentist's responsibility to design the denture supporting area on some accurate models and it is outside the scope of this section to expand on this aspect of full denture construction other than to say that it should not be left to the technician.

\section{Determining the interarch relationship 'bite registration'}

The term 'bite registration' is a poor one; as the patient is not asked to bite into anything; in fact if they do, it is likely that they will make an uncontrolled mandibular movement away from CR.

The purpose of this stage is to record the relationship between the upper and lower jaws; in the vertical, horizontal and anterio-posterior planes. Before this stage can be completed, the decision whether to make the dentures to the conformative or re-organised approach must have been taken. In addition, it must have been decided whether only a balanced static occlusion (balanced occlusion) or also a balanced dynamic occlusion (balanced articulation) is
The provision of full dentures will involve three stages:

\section{Colloquial term Actual function}

I. 'impression making' designing of the fitting surfaces

2. 'bite registration' determining the interarch relationship

3. 'setting up the teeth' providing the ideal occlusion

needed. If balanced articulation is indicated a facebow record must be taken so that the occlusal rims can be mounted into a semiadjustable articulator.

\section{Gothic arch trace}

One means by which a balanced articulation is created on that articulator is the gothic arch trace. This trace is made on a 'central bearing apparatus' (Fig. 11a). This comprises upper and lower acrylic plates onto which is mounted centrally, a stylus and a platform.

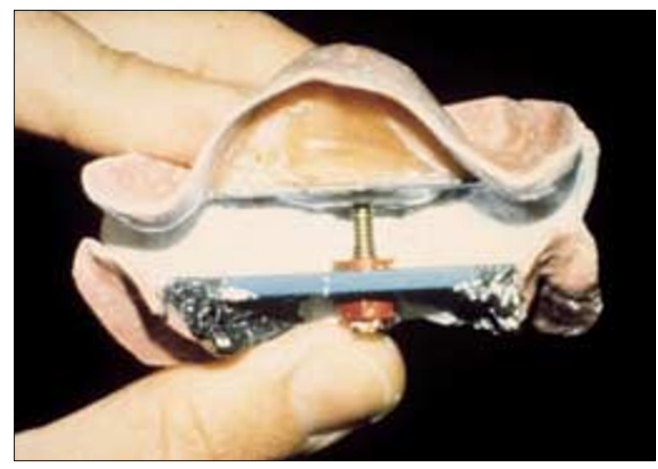

Fig I la Gothic arc trace apparatus

These will record a 'map' of the patient's range of movements, by asking the patient to go into:

- Protrusive

- Right lateral excursions.

- Left lateral

The starting point of these movements as inscribed by this trace is the arrowhead (Fig. 11b) and represents centric relation (CR or RCP). If it proves impossible to obtain an arrowhead, this means that the patient does not have a reproducible maxillo-mandibular relationship. This is an important finding and would indicate the need for some further predefinitive treatment in order to discover a reproducible jaw relationship (ie CR).

This could be achieved by the use of 'pivotal appliances'. A polished pivotal appliance (Fig.12) may look unusual but it is remarkable how well they are tolerated. After fitting, further adjustments are easily made to find the 
Fig I I b Gothic arc trace arrowhead

Fig I 2 Registering CR with a pivotal appliance

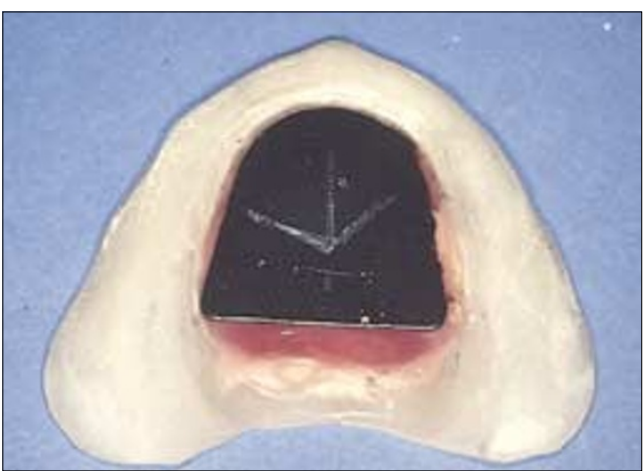

new occlusal vertical dimension (OVD) and to provide occlusal stability. When all adjustments have been made and the patient has been wearing the appliance comfortably for a period, it is a simple procedure to register centric relation with a registration material placed before and behind the pivots. Pivotal appliances may be made on acrylic bases, or from the patient's previous dentures. It may be wise to make a copy of the patient's denture with which to make the pivotal appliance. It will then be possible to return the original denture intact to the patient.

\section{Providing the ideal occlusion}

'Setting up the teeth'

The occlusion of the dentures that will be 'ideal' for the patient is the one which will limit the tilting of the dentures and so minimalise disruption to the peripheral seal, risking instability. As stated this occlusal prescription will take into account the patient's denture bearing tissues and their chewing pattern.

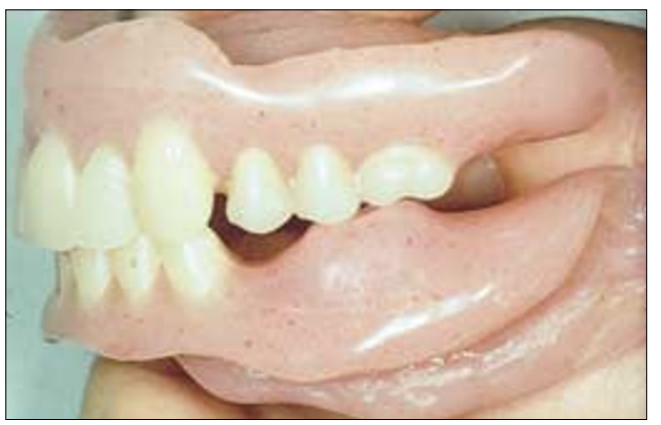

On the basis of occlusal form, there are four types of posterior teeth: ${ }^{8}$

- Anatomic teeth: these 'duplicate' the anatomical form of natural teeth and typically have $30^{\circ}$ to $40^{\circ}$ cuspal angles. Modified forms have $20^{\circ}$ cuspal angles and these are typically used in complete dentures, on the basis that it is easier to obtain balanced articulation with $20^{\circ}$ cuspal angles.

- Non-anatomic teeth: these have occlusal surfaces that are not anatomically formed and are designed with mechanical and not anatomical principles in mind.

- Zero-degree teeth and

- Teeth without cusps: these may be used for patients who have essentially vertical chewing movements as only their static occlusion needs to be balanced (balanced occlusion), ie there is no need for a balanced dynamic occlusion (balanced articulation).

In those patients who need a balanced dynamic occlusion, a cuspal form is essential. The dentist has to consider what is appropriate for the patient. If large ridges are present, anatomical teeth will probably suffice. Whereas, if the ridges are flat or where implant-supported dentures oppose maxillary complete dentures, then teeth with nonanatomical cuspal form may be indicated ${ }^{8,9}$ as large cusps may induce instability via a tripping effect.

The EDEC principle in complete denture construction $(\mathrm{E}=$ Examine, $\mathrm{D}=$ Design, $\mathrm{E}=$ Execute, $\mathrm{C}=$ Check)

The construction of complete dentures is a sequential process. The successful completion of one stage is the prerequisite of starting the next. In order to prevent introducing errors into the sequence it is important to consider what each stage needs to achieve. The EDEC principle is one way of defining the objectives of the process.

\section{$\mathrm{E}=$ Examine}

The examination of the denture bearing surfaces, the existing dentures and the masticatory pattern will be the basis for

$\mathrm{D}=$ the design of the occlusion of the prosthesis.

The first stage of full denture construction is: $\mathrm{E}=$ the execution of that design. This comprises the procedures up to and including the jaw registration. It involves defining the relationship between the denture bearing surfaces in three planes and the design of the ideal static and dynamic occlusion for that patient. At one end of the spectrum this design may be a copy of the patient's previous prosthesis: 'the conformative approach'. Alternatively, there may be need to design changes in the vertical height, jaw relationship and occlusal prescription: 'the re-organised approach'. The design will be achieved and recorded in the jaw registration, when this information is passed to the laboratory technician. From that moment the emphasis of the dentist, during the try-in and completion stages, moves from designing and executing change to

$\mathrm{C}=$ checking that there is no deviation from the record supplied to the technician; ie from the bite registration to completion: 'the conformative approach' 


\section{Combination syndrome ${ }^{10}$}

The final section under the heading of 'mucosa supported dentures' considers the type of occlusion that is required when a full denture is opposed by teeth or a fixed prosthesis, a condition referred as the 'Combination Syndrome. ${ }^{10}$

There are three scenarios:

1. Complete maxillary denture opposed by dentate/partly dentate mandibular arch.

2. Complete mandibular denture opposed by dentate/partly dentate maxilla

3. Complete maxillary denture opposed by implant retained lower complete denture ('New Combination Syndrome').

Complete maxillary denture opposed by dentate/partly dentate mandibular arch In this situation, these can be considerable displacing forces on the upper denture resulting from mandibular movements, so the retention of the upper denture must be maximised.

Displacing forces can be reduced by co-ordinating the maxillary teeth and maxillary plane of occlusion to mandibular movement. This is achieved by:

- Using a facebow to transfer the plane of the upper arch to the condylar axis.

- Using a central-bearing screw to create an arrowhead (gothic arch) tracing.

- Setting the articulator condylar angles to accord to the border tracings on the arrowhead tracing.

- Establishing, carefully, at trial insertion, that CR (RCP) is reproducible.

- 'Milling in' the occlusion to suit the patient. This will inevitably be necessary, as cuspal inclines of the denture teeth will be unlikely to be equal to those of the patient's natural mandibular teeth.

- Reviewing of the patient after 3 days to refine the cuspal anatomy.

Complete mandibular denture opposed by dentate/partly dentate maxilla

Success is even more difficult to achieve in this clinical scenario than the former and although similar techniques are recommended, two major problem areas are often present:

- Impaired support potential of the mandibular denture-bearing tissues

- Unfavourable peri-denture anatomical forces, ie muscle attachments.

Complete maxillary denture opposed by implant retained lower complete denture 'New Combination Syndrome'

This third scenario is now encountered with increasing frequency. This application of implants is rightly considered for the patient who is suffering because of their unretentive and uncontrollable lower complete denture. But dentists should be aware of the possibility of the patients developing a 'New Combination Syndrome'.

At its simplest this syndrome will clinically present as ineffective mastication, often associated with a very unretentive upper denture. At its most extreme the patient may exhibit periods when they are unable to exert any control over their mandible or find any position where they can rest it, exhibiting a severe mandibular tremor.

The hypothesis on how this syndrome arises is as follows:

As the lower denture becomes more retentive:

- It is known that there is a significant increase in displacing forces transmitted to the upper denture by virtue of the increased retention afforded to the lower denture by the mandibular implants. This force may be considerably in excess of the retention that has been provided to the upper denture, a retention that was perfectly acceptable when it was opposed only by a unretentive lower denture on maybe an atrophic ridge.

- It is thought that there may be a significant change in the patient's chewing pattern and that this might occur some time after the implant retained lower prosthesis has been fitted.

Before implants were used, the patient probably developed a purely vertical chewing pattern in an attempt to accommodate to the extremely unretentive lower denture. Once the lower denture is retained by the implants, however, the patient may revert back to a masticatory pattern that includes lateral and protrusive movements (the ruminatory pattern of mastication). The occlusion of the dentures, which was acceptable whilst the patient was chewing only with vertical movements, could now be 'tripping' the upper denture.

\section{Good occlusal practice is different in the edentulous patient from that in the dentate patient. \\ The form of an ideal occlusion for a particular edentulous patient will depend on their chewing pattern and ridge form}

Complete denture construction is a sequential process:

- Examination

$$
\begin{aligned}
& \text { denture bearing surface } \\
& \text { existing dentures } \\
& \text { masticatory pattern }
\end{aligned}
$$

- Design the appropriate occlusion

- Execute that design

(up to and including jaw registration)

- Check that there is no deviation from that design (from registration to completion) 


As the chewing
pattern
changes, the
features of the
ideal occlusal
prescription
also change

1. Picton D C A, Wills D J. Viscoelastic properties of the periodontal ligament and mucous membrane. J Prosthet Dent 1990; 40: 263-272.

2. Applegate O C. Essentials of Removable Partial Denture Prosthesis. pp166-194. Philadelphia: WB Saunders Co, 1954.

3. Applebaum M. Plans of Occlusion. Dent Clin N Amer 1984; 28: 273-276.

4. Jacobson T E, Krol A J A. A contemporary review of the factors involved in complete denture retention, support and stability. J Prosthet Dent 1983; 49: 5-15; 165-172, 306-313.

5. British Society for the Study of Prosthetic Dentistry. Guidelines in Prosthetic and Implant Dentistry. pp29-47. London: Quintessence Publishing Co. Ltd, 1996.

6. McCord J F, Grant A A. A Clinical Guide to Complete Denture Prosthetics. London: BDJ Books, 2000.

7. Moses $\mathrm{CH}$. Physical considerations in impression making. J Prosthet Dent 1953; 3: 449-463.

8. Lang B R. Complete Denture Occlusion. Dent Clin N Amer 1996, 40: 85-101.

9. Denisen H W, Kalk W, van Wass M A J, van Os J H. Occlusion for maxillary dentures opposing osseointegrated mandibular prostheses. J Prosthet Dent 1993; 6: 446-450.

10. Kelly E. Changes caused by a mandibular partial denture opposing a maxillary complete denture. J Prosthet Dent 1972; 27: 140-150.

\section{Solutions}

Some of the solutions include:

- The basic principles of good retention and stability are not only still needed, they are more important.

- The occlusion should be designed to reduce the displacing or 'tripping' forces, even in a patient who appears at the time of examination to have a vertical masticatory pattern ('accommodative chomping').

- The possibility that the patient will need some implants on the upper jaw should be raised, ideally before the implant treatment of the lower jaw is finalised.

\section{Treatment strategies and summary}

The principle is to design and provide an occlusion that is ideal - ideal for the important criteria of denture success - stability. An occlusion is needed that will reduce the displacing forces on the denture(s).

The basic equations are:

$$
\begin{aligned}
& \text { Force }>\text { Retention }=\text { Instability } \\
& \text { Force }<\text { Retention }=\text { Stability }
\end{aligned}
$$

The occlusal prescription provided is a major factor in determining the size of the force applied to the dentures. The retentive capacity of the denture is defined by the patient's tissue and masticatory patterns.

$$
\begin{aligned}
& \text { Force }>\text { Retention }=\text { Instability } \\
& \text { Force }<\text { Retention }=\text { Stability }
\end{aligned}
$$

\section{Guidelines of good occlusal practice}

I The examination of the patient involves the teeth, periodontal tissues and articulatory system.

2 There is no such thing as an intrinsically bad occlusal contact, only an intolerable number of times to parafunction on it.

3 The patient's occlusion should be recorded, before any treatment is started.

4 Compare the patient's occlusion against the benchmark of ideal occlusion.

5 A simple, two dimensional means of recording the patient's occlusion before, during and after treatment is an aid to good occlusal practice.

6 The conformative approach is the safest way of ensuring that the occlusion of a restoration does not have potentially harmful consequences.

7 Ensuring that the occlusion conforms (to the patient's pre-treatment state) is a product of examination, design, execution and checking (EDEC)

8 The 'reorganised approach' involves firstly the establishment of a 'more ideal' occlusion in the patient's pretreatment teeth or provisional restorations; and then adhering to that design using the techniques of the 'conformative approach'

9. An 'ideal occlusion' in removable prosthodontics is one which reduced de-stabilisating forces to a level that is within the denture's retentive capacity. 